\title{
Drama und Theater bis 1933
}

\author{
Georg-Michael Schulz
}

„Mit der Aufklärung, mit Moses Mendelssohn, beginnt die deutsch-jüdische Literaturgeschichte, insofern er durch seine Bibelübersetzung und seinen Bibelkommentar dem jüdischen Publikum die deutsche Sprache nahezubringen und die Bibel im Geist der Aufklärung verständlich zu machen suchte“ (Horch und Shedletzky 1992, 291). In dem besonderen Fall der Gattung „Drama“ ist es indessen nahe liegend, Gotthold Ephraim Lessing an den Anfang zu setzen, der bereits in seinem frühen Lustspiel Die Juden (ersch. 1754) antijüdische Vorurteile anprangert und der besonders in seinem Schauspiel Nathan der Weise (1779) dem Judentum einen zentralen Stellenwert einräumt. Der edle Nathan, den Lessing mit Zügen seines Freundes Moses Mendelssohn ausstattet, vertritt hier die aufklärerische Leitvorstellung der Toleranz, indem er mit der berühmten Ringparabel auf die ihm gestellte Frage antwortet, welche der drei Religionen Judentum, Christentum, Islam die „wahre“ sei. Der Parabel zufolge ist die „Wahrheit“ einer Religion nicht argumentativ nachweisbar, sie muss in der Lebenspraxis der jeweiligen Gläubigen zum Vorschein kommen. Die Gläubigen sollen durch Ergebenheit in Gott und soziale Tugenden wie Sanftmut, Verträglichkeit, Wohltätigkeit die Wahrheit ihrer jeweiligen Religion zu Tage treten lassen. Das Stück endet (der Bühnenanweisung zufolge) „mit stummer Wiederholung allseitiger Umarmungen" und besitzt in der gelingenden Verständigung und Versöhnung etwas Utopisches. In der Wertschätzung der Juden erlangt Lessing im Laufe des 19. Jahrhunderts jedenfalls ein hohes Renommee, wenngleich es dann auch kritischere Stimmen gibt. So erfahren Lessing und Mendelssohn, beide Jahrgang 1729, anlässlich ihres gemeinsamen Jubiläums im Jahr 1929 recht unterschiedliche Beurteilungen zum Beispiel von dem Schriftsteller Arnold Zweig und dem Pädagogen Ernst Simon. Während Zweig mit Blick auf Mendelssohn und unter Einbeziehung auch Lessings den offenen Horizont der Aufklärung, das Walten von Verstand und Einsicht preist, kann Simon nichts eigentlich Jüdisches in Lessings Nathan erkennen, in einer idealisierten Figur, die aber gleichwohl vielen sich assimilierenden Juden als Vorbild gedient habe, während andererseits Mendelssohn zum „Großvater des Taufjudentums" geworden sei (vgl. Horch 1999, 50-51).

Goethe und Schiller, wiewohl ihrerseits nicht weiter am Judentum, sondern allenfalls an den biblischen Texten interessiert, werden dennoch im 19. Jahrhundert für viele Juden zu Leitbildern, nicht zuletzt aufgrund der Humanitätsvorstellungen der Weimarer Klassik. Unter den Romantikern indessen gibt es eine verbreitete Aversion gegenüber den Juden. Das zeigt sich unverhohlen etwa in Achim von Arnims Doppeldrama Halle und Jerusalem (1811), in dessen erstem Teil ein reicher jüdischer Händler (in der Szene II/5) erzählt, er sei von den Juden in Jerusalem um Geld gebeten worden, damit sie die von den Türken gefangen gehaltenen Glaubens- 
brüder freikaufen können. Der Händler meint jedoch, das gehe ihn nichts an. Und in anderem Zusammenhang erklärt er, dass der auf seinem Volk ruhende Segen Gottes durch die Taufe nicht abgewaschen werden könne. Diesem Händler hat Arnim den Namen „Nathan“ gegeben (vgl. Lea 1978, 15-18).

Während in den (literarisch anspruchsvolleren) Dramen der Aufklärung überwiegend positive jüdische Figuren auftreten, erscheinen Juden auf den Theaterbühnen nicht selten als komische Figuren (vgl. Bayerdörfer 1989, 94-95). Im frühen 19. Jahrhundert begegnet diese karikaturistische Tendenz dann etwa in den Stücken von Julius von Voß: Der travestirte Nathan der Weise. Posse in zwei Akten mit Intermezzos, Chören, Tanz, gelehrtem Zweykampf, Mord und Todschlag, auch durch Kupfer verherrlicht (1804). Darin wird die „Rückstufung des Nathan auf den mauschelnden Schacherjuden“ (Bayerdörfer 1989, 104) ergänzt durch die Karikierung der Assimilationsbereitschaft Rechas, der Tochter Nathans. Karl Borromäus Alexander Sessa treibt dann in seiner - tatsächlich erfolgreichen und quasi „Schule“ machenden - Posse Unser Verkehr (1815) die Klischierung noch weiter: Es treten fast nur Juden auf, und für die gibt es nur einen einzigen Wert: das Geld; das gilt zu guter Letzt auch für die jüngeren Figuren, die zwar bemüht, aber nicht wirklich fähig sind, sich zu assimilieren (vgl. Neubauer 1987). Voß wiederum sieht sich durch diese Posse angeregt, ein „Gegenstück“ (Euer Verkehr) zu schreiben, in dem vor allem Schauspieler, die Sessas Posse zeigen wollen, lächerlich gemacht werden, und veröffentlicht noch zahlreiche weitere Stücke mit Judenfiguren (vgl. Neubauer 1994, 189-190). Wenngleich Voß - dem Vorwort zum Travestirten Nathan zufolge - sich nicht als einen der „Feinde“ der Juden sehen will, so läuft seine effektorientierte Fixierung auf Juden als komische Figuren indessen doch auf deren Abwertung hinaus. Damit ist eine Entwicklung in Gang gesetzt, in deren Verlauf die für die Aufklärung charakteristische Umwertung der Judenfiguren ins Positive „in allen Punkten rückgängig gemacht“ wird (Bayerdörfer 1989, 108).

Ein durchaus erfolgreicher Dramatiker und Prosaautor ist Ludwig Robert, ein Bruder Rahel Varnhagens. Sein erfolgreichstes Drama, Die Macht der Verhältnisse, von 1815 an in etlichen Theatern aufgeführt, behandelt einen Konflikt zwischen einem Grafen und einem Bürger, der die Ehre seiner Schwester vor dem Grafen schützen will. Der Graf lehnt eine Duellforderung des Bürgers ab, der ihn daraufhin erschießt und, zum Tode verurteilt, sich das Leben nimmt. Verarbeitet und in eine Art „bürgerliches Trauerspiel“ transponiert mit der dafür charakteristischen Ständedifferenz Adel / Bürgertum ist hier die Itzig-Arnim-Affäre (1811): Im Salon der Sarah Levy geraten deren Neffe Moritz Itzig und Achim von Arnim aneinander. Eine daraus sich ergebende Duellforderung Itzigs lehnt Arnim ab mit dem Hinweis, ein Jude sei nicht satisfaktionsfähig. Daraufhin überfällt Itzig Arnim, wogegen dieser sich freilich zu wehren vermag (vgl. Lea 1978, 19-22). 1813 stirbt Itzig als preußischer Soldat in den Befreiungskriegen gegen Napoleon. 
Bereits vorher, 1811, ist Roberts Tragödie Die Tochter Jephthas (nach einer Erzählung aus dem biblischen Buch der Richter) in Breslau uraufgeführt und noch im selben Jahr von Goethe auch in Weimar auf die Bühne gebracht worden. Das Stück bemüht sich, den biblischen Stoff in einer psychologisch differenzierten Weise nahezubringen, einen Stoff, der vielfach in musikalischen (Händel, Meyerbeer) und literarischen Werken (zuletzt noch Feuchtwanger) behandelt worden ist: Der Feldherr Jephtha gelobt, im Fall eines Sieges das, was ihm bei seiner Heimkehr zuerst begegnen werde, $\mathrm{zu}$ opfern; das ist dann seine Tochter Dina, die ihrer Opferung zustimmt (vgl. Weissberg 1996).

Ludwig Börne und Heinrich Heine sind zweifellos herausragende Vertreter der deutsch-jüdischen Literatur des 19. Jahrhunderts, nicht jedoch der Dramengeschichte. Denn Börne hat zwar Theaterkritiken geschrieben, aber keine Dramen, und auch für Heine ist das Drama keine bevorzugte Ausdrucksform. Zu nennen ist immerhin seine Tragödie Almansor (1820-1822), die von einer unglücklich verlaufenden Liebesgeschichte handelt, und zwar im Zusammenhang mit der christlich-islamischen Auseinandersetzung in Spanien, die das eigentliche Thema des Stücks ist. Die Tragödie spielt kurze Zeit, nachdem die Mauren 1492 besiegt worden sind und nur dann in Spanien bleiben dürfen, wenn sie den christlichen Glauben annehmen. Heines Sympathie gehört ganz unzweifelhaft nicht den Christen, sondern den Mauren, auch den zum Christentum übergetretenen. Für den christlichen Standpunkt steht unter anderem der (die Inquisition erneuernde) Kardinal Ximenes. Als dieser einen Koran verbrennt, fallen die inzwischen oft zitierten Worte: „Das war ein Vorspiel nur, dort wo man Bücher / Verbrennt, verbrennt man auch am Ende Menschen.“ (Heine 1975, 284-285)

Die Juden spielen in dem Stück zwar keine Rolle, aber selbstverständlich hat Heine sie durchaus im Blick, denn gerade auch sie, die Sepharden, werden im selben Jahr 1492 ebenfalls aus Spanien vertrieben (und 1531 nach der Einführung der Inquisition auch aus Portugal), sofern sie nicht zum Christentum konvertieren.

Nur beiläufig erwähnt sei Christian Dietrich Grabbes Drama Aschenbrödel (1835), in dem - als komische Figur - ein jüdischer Geldverleiher vorkommt, der alle möglichen albernen Versuche unternimmt, sein Geld wiederzubekommen, und am Ende immerhin entschädigt wird.

Während Karl Gutzkow in König Saul (1839) anhand eines biblischen Stoffs Fragen der Macht und unterschiedliche menschliche Haltungen behandelt, zeigt er in Uriel Acosta (1846) einen Amsterdamer Juden, der aufgrund seiner abweichenden religiösen Überzeugungen in einen Konflikt mit der Orthodoxie gerät, einen auch noch um persönliche Beziehungsprobleme angereicherten Konflikt, an dem er zerbricht. Gutzkow zeichnet darin ein ausgewogenes Bild des Judentums ohne stereotype Züge, sodass die Angriffe auf die Orthodoxie ohne Weiteres auch als auf das Christentum gemünzt gesehen werden können.

In zwei Dramen Franz Grillparzers besitzen Jüdinnen eine dominierende Rolle, aber eher als individuelle Persönlichkeiten und weniger aufgrund ihrer Zugehörig- 
keit zum Judentum, nämlich die impulsiv-unbesonnene, verführerische Rahel in der Tragödie Die Jüdin von Toledo (1851) und Esther in dem fragmentarischen gleichnamigen Drama, eine durch ihre moralische Geradlinigkeit und Klugheit beeindruckende Figur. Während das Judentum in Esther rein positiv erscheint, repräsentiert durch Esther und zudem noch durch Mardochai, ihren rigoros rechtlich gesonnenen Oheim, enthält Die Jüdin von Toledo auch anders getönte Momente; nachdem Rahels Stern gestiegen ist, zeigt ihr Vater, zu einer gehobenen Position gelangt, einen recht hartherzigen Umgang mit mittellosen Bittstellern.

Auf das apokryphe biblische Buch Judith greift Friedrich Hebbels gleichnamiges erstes Drama (1840) zurück. Es geht darin um zwei außerordentliche Menschen, Holofernes, den Heerführer des babylonischen Königs Nebukadnezar, und Judith, eine junge Witwe aus der jüdischen Stadt Bethulien. Holofernes, tyrannisch-grausam, unberechenbar und selbstgefällig, bedroht Bethulien, woraufhin, da die männlichen Einwohner versagen, die mutig-leidenschaftliche Judith sich zu einer außerordentlichen Tat veranlasst sieht: Sie geht ins Lager des Holofernes, wird von diesem vergewaltigt und enthauptet ihn anschließend. Erotische Motive und religiöse Impulse bestimmen in einer komplex-widersprüchlichen Verbindung von freien Willensakten und Notwendigkeiten insbesondere Judiths Handeln. Die jüdische Geschichte und die jüdische Religiosität bilden nur die Grundlage, nicht das eigentliche Thema des Dramas.

Nicht ein biblisches Buch, sondern die jüdische Geschichte, wie sie der Geschichtsschreiber Josephus erzählt, liefert die Grundlage für Herodes und Mariamne (1848). Der König Herodes kann den Gedanken nicht ertragen, seine Frau Mariamne könnte nach seinem Tod einen anderen Mann heiraten, und befiehlt daher heimlich ihre Tötung für den Fall seines Todes. Mariamne, von vornherein gewillt, ebenfalls zu sterben, falls Herodes sterben sollte, erfährt von diesem Befehl und ist tief gekränkt. Emotionale Verhärtungen auf beiden Seiten führen schließlich dazu, dass Herodes Mariamne des Ehebruchs verdächtigt und sie töten lässt, bevor er erfährt, dass Mariamne vollkommen unschuldig gewesen ist. Wenn am Ende noch - etwas fremdartig - die Heiligen drei Könige auftauchen auf der Suche nach dem neugeborenen „König der Könige“ (was Herodes veranlasst, den bethlehemitischen Kindermord anzuordnen), dann ist das ein Hinweis auf das Christentum als eine historisch spätere Epoche; es schließt keine Auseinandersetzung mit Judentum und Christentum als geistigen Größen ein.

Am Rande erwähnt sei noch Hebbels Komödie Der Diamant (1847), in der ein Jude einen ihm nicht gehörenden Diamanten verschluckt, hinter dem nun alle Welt her ist und der noch rechtzeitig auf natürlichem Wege wieder zu Tage tritt, bevor die Drohung, dem Juden den Bauch aufzuschneiden, wahr gemacht wird.

Selbstverständlich gibt es in der ersten Hälfte des 19. Jahrhunderts noch weitere Behandlungen biblischer Stoffe durch weniger bekannte Autoren, darunter auch jüdische Autoren, wie etwa Karl Beck mit seinem Drama Saul (1841; vgl. Lea 1978, 
41-48). Nur entlehnt ist der biblische Name dagegen in Salomon von Mosenthals sehr erfolgreichem ,Volksschauspiel‘ Deborah (1849), und zwar entlehnt für eine ungarische Jüdin im ausgehenden 18. Jahrhundert, die gegen ihre herkunftsbedingte Außenseiterposition rebelliert und damit einen gewissen Erfolg erzielt (vgl. Lea 1978, 62-69).

In einer ganz anderen Weise, nämlich in einer Parodie, begegnet eine bereits erwähnte biblische Gestalt nochmals: Hebbels Judith kehrt wieder in Johann Nestroys Posse Judith und Holofernes, einer Posse, die zunächst einmal das Hebbelsche Drama parodiert. Welche Haltung gegenüber den Juden diese Posse einnimmt und welche Wirkung sie hat, ist schwer zu ermitteln und wird daher auch in der Forschung unterschiedlich beurteilt. Während übrigens im Wiener Volkstheater vergleichsweise wenige jüdische Figuren auftreten, häufig (ohne eine bestimmte Tendenz) ebenso behandelt wie „bodenständige Wiener Typen“ (Hein 1988, 171), „wimmelte es“ andernorts im „populären Bühnendrama“ der ersten Hälfte des 19. Jahrhunderts geradezu „von Judenfiguren“, „durchweg ins Ideale emporstilisiert bzw. ins Gemeine herabgewürdigt“ (Denkler 1988, 149). Einen Beitrag dazu leistet auch der erfolgreiche jüdische Possenautor David Kalisch, der vielfach als „Vater der Berliner Posse“ eingestuft wird und jüdische Figuren meist eher mit ein wenig Spott behandelt und sie nur gelegentlich auch sehr positiv herausstellt (Einer von unsere Leut', 1859).

Dabei muss man sich vergegenwärtigen, dass die Texte nur eine eingeschränkte Vorstellung vom Erscheinungsbild jüdischer Figuren auf der Bühne vermitteln, selbst wenn die Figuren, den Texten zufolge, das „Juden-Deutsch“ sprechen als eine (mehr oder minder konstruierte) sprachliche Variante, die die Herkunft des Sprechers offenbaren soll. Proxemik, Gestik und Mimik, an „stereotypen Mustern der komischen Figur orientiert“ (Bayerdörfer 1989, 100), kommen hinzu und spielen eine wichtige Rolle bei der erneuten Verfestigung antijüdischer Klischees, wie sich anhand von Berichten und Illustrationen zeigen lässt (vgl. Bayerdörfer 1989, 100-102, 109 u.a.).

Nachdem in der zweiten Hälfte des 19. Jahrhunderts Romane und Erzählungen im Vordergrund gestanden haben, erlebt das Drama als literarische Gattung mit dem Beginn der Moderne um 1890 einen Aufschwung. Das gilt für die >Berliner Moderne` ebenso wie für die >Wiener Moderne . Während Juden in Berlin diesen Aufschwung vor allem in den Medien (Verlagen, Theatern, Presse - man denke an den bedeutenden Kritiker Alfred Kerr) sowie nicht zuletzt als Rezipienten mittragen, gibt es in Wien auch einige jüdische Autoren als Mitwirkende, deren Darstellungen jüdischer Figuren sich hinsichtlich der Ernsthaftigkeit und der Differenziertheit deutlich von der Typisierung der Figuren in den Possen und den spopulären Dramen abheben. Richard Beer-Hofmann lässt in seinem Trauerspiel Der Graf von Charolais (1904) einen hartherzigen jüdischen Geldverleiher auftreten, der seine Haltung in einer 
beeindruckenden großen Rede als folgerichtiges Ergebnis der immer schon erfahrenen Missachtung von Juden durch die nicht-jüdische Umgebung erklärt. Der Regisseur der Uraufführung 1904 in Berlin, Max Reinhardt, spielte selbst den jüdischen Geldverleiher und erhielt „bei offener Bühne stürmischen Applaus“ (Kindermann 1968, 315). Beer-Hofmanns Hauptwerk ist der unvollendete Dramenzyklus Die Historie von König David (1898-1937), der von dem Motiv des Auserwähltseins des jüdischen Volkes und des fortbestehenden Bundes zwischen Gott und seinem Volk ausgeht und in einer nachgerade sepischen` Entfaltung der biblischen Welt eine geschichtlich-heilsgeschichtliche Perspektive entwickelt entgegen den historischen Erfahrungen des Judenhasses und des gerade auch nach dem Ersten Weltkrieg virulenten Antisemitismus.

In der letzteren Hinsicht setzt Professor Bernhardi (1912) ganz andere Akzente, eine Komödie Arthur Schnitzlers, der mit seinen Dramen seit Mitte der 1890er Jahre (Liebelei, 1895) Erfolge verzeichnet: Als der jüdische Arzt und Klinikdirektor Bernhardi einem katholischen Priester den Zutritt zu einer Sterbenden verwehrt, um diese zu schonen, wird der Vorfall von antisemitisch gesonnenen Gegnern (und den Antisemitismus zum eigenen Vorteil nutzenden Konkurrenten) Bernhardis aufgebauscht und hat schließlich als „Störung des Religionsfriedens“ eine zweimonatige Gefängnisstrafe für Bernhardi zur Folge, der freilich am Ende seine berufliche Position wiedererlangt. Eine persönliche Aussprache zwischen ihm und dem Priester zeigt die bleibende Differenz zwischen verschiedenen Haltungen, denen einerseits das ärztliche Ethos und andererseits die Rücksicht auf das Wohl der Kirche zugrunde liegen. Aber unter dieser immerhin noch rationalisierbaren Differenz liegt eine im „inneren Gefühl“ fortbestehende Kluft von „höherer“ und „hoffnungsloserer“ Art (4. Akt), die auch nicht durch einen versöhnlichen Händedruck zu schließen ist, die Kluft zwischen dem Juden und dem Nicht-Juden. Im Übrigen präsentiert das Drama ein bemerkenswert reiches Spektrum an Haltungen mittels einer ganzen Palette von unterschiedlich nuancierten Nebenfiguren. Seine Uraufführung erlebt das Drama im November 1912 in Berlin, übrigens am selben Abend, an dem Otto Brahm stirbt, seinerseits jüdischer Herkunft und ehedem der Schöpfer des naturalistischen Bühnenstils (vgl. Brauneck 1999, 679-680). In Österreich ist das Drama indessen noch bis 1918 mit einem Aufführungsverbot belegt, weil es staatliche Einrichtungen in entstellter Weise vorführe (vgl. Beier 2008, 218-220).

An der Weiterentwicklung der Gattung des Dramas nach dem Naturalismus haben jüdische Autoren einen beachtlichen Anteil. Dabei sind viele ihrer Stücke zwar inhaltlich und formal bedeutsam und wichtig in literaturgeschichtlicher Hinsicht, aber sie haben nicht selten wenige oder gar keine Bezüge zum Judentum. In die mythischen Weiten der Weltschöpfung greift Aeon (1907-1911) hinaus, Alfred Momberts - von Martin Buber geschätzte - Trilogie von ekstatisch-visionären VersDramen. Andere Stücke behandeln menschlich-existenzielle Fragen, so etwa Franz Werfels Dramen Die Troerinnen (1915) und Der Spiegelmensch (1920) oder Paul Korn- 
felds Stück Die Verführung (1916), und sie tun dies zum Teil in dem emphatischgesteigerten Stil des Expressionismus. Weitere Dramen liefern Bilder der Gesellschaft, dies zumindest ausschnitthaft, etwa Else Lasker-Schülers Schauspiel Die Wupper (1909), oder - in weiter ausgreifender Weise - Carl Sternheims Komödien und Schauspiele, die zum Teil unter dem Sammeltitel Aus dem Bürgerlichen Heldenleben (1910-1922) zusammengefasst sind. Sie bemühen sich mit dezidiert sozialkritischer Intention, die Verlogenheit des Bürgertums durch dessen Sprache zu entlarven. Nebenbei begegnet in dem ersten dieser Stücke, Die Hose (1910), auch einmal eine Figur wie der für Richard Wagner schwärmende Friseur Benjamin Mandelstam, der seine zuvor in Abrede gestellte jüdische Herkunft durch den Ausdruck „koscher“ verrät. Sternheims Stücke sind im Übrigen fast die einzigen aus dem ,expressionistischen Jahrzehnt“, die sich dauerhaft im Theaterrepertoire etabliert haben.

Im Expressionismus konvergieren gleichsam die Hoffnungen auf Erneuerung und auf Gemeinschaft im Geistigen, nämlich die Hoffnungen der gesellschaftlich ausgegrenzten Juden wie der isolierten Intellektuellen und Künstler. Das zeigt etwa Ernst Tollers Stück Die Wandlung (1919), in dessen Zentrum ein jüdischer Kriegsfreiwilliger steht, ein Bildhauer, der durch seinen Patriotismus und seine Kriegsteilnahme die Anerkennung als Deutscher erringen möchte, der sich dann aber, durch seine Kriegserlebnisse desillusioniert, zum Pazifisten wandelt und sich gegen jeden Nationalismus wendet. Einen großen Erfolg hat die Uraufführung mit Fritz Kortner in der Hauptrolle, nicht zuletzt weil sie sich in der Inszenierung von Karl Heinz Martin um einen neuen Bühnenstil, einen expressionistischen Andeutungsstil, bemüht (vgl. Englhart 2008).

In anderer Weise ebenfalls um den Ersten Weltkrieg geht es dem Wiener Kulturund Sprachkritiker Karl Kraus in seinem Mega-Drama Die letzten Tage der Menschheit (1915-1921) mit 220 Szenen (auf mehr als 600 Seiten) und mit einer riesigen Zahl von Personen aus allen gesellschaftlichen Schichten unter Einschluss von Personen der Zeitgeschichte. Kraus zielt darin auf die satirisch-polemische Entlarvung der für den Krieg verantwortlich gemachten Haltungen der Borniertheit, der Inkompetenz, der Anmaßung, der Lüge, der Korruption, der Brutalität usw., Haltungen, für deren Förderung Kraus insbesondere die Presse und deren korrumpierte Sprache verantwortlich macht (wobei seine Kritik auch Juden und Jüdisches mit einbezieht). Daher legt er besonderen Wert auf authentische Zitate: „Die unwahrscheinlichsten Gespräche, die hier geführt werden, sind wörtlich gesprochen worden; die grellsten Erfindungen sind Zitate“, heißt es im Vorwort. Erst 1964 kam das Stück in einer gekürzten Fassung in Wien zur Uraufführung.

So breit das Spektrum der Dramen-Themen ist, die von jüdischen Autoren behandelt werden, darf indessen nicht übergangen werden, dass etliche dieser Autoren in denselben Jahren auch mit dezidiert jüdischen Themen ans Licht der Öffentlichkeit treten. Das gilt etwa für Arnold Zweig, der dies gleich in drei Dramen tut. Abigail und Nabal (1913) zeigt im Rückgriff auf einen biblischen Stoff, wie Abigail 
und der spätere König David, hernach ihr Mann, einander nahe kommen, und präsentiert - ganz in der Art des Expressionismus - mehrere seelisch zerrissene Figuren: „Die Brust speit Worte wie Blitze aus Wolken“ (Szene III, 4). - Die Sendung Semaels (1918, überarb. 1920, ursprüngl. unter dem Titel Ritualmord in Ungarn 1914) verbindet zwei sehr unterschiedliche Szenen-Komplexe: einerseits die realistische Darstellung einer historischen Begebenheit, nämlich einer antijüdischen Volksempörung aufgrund eines angeblichen Ritualmords an einem in Wirklichkeit ertrunkenen Mädchen im ausgehenden 19. Jahrhundert in Ungarn, und andererseits dazwischen geschobene Szenen in einem außerirdischen Bereich, in denen Gott als „die Stimme des Elohim“ zu vernehmen ist und in denen außer Semael, dem Teufel, mehrere biblische und historische Gestalten auftreten. Die „Sendung“ Semaels besteht darin, durch sein zerstörerisches Wirken die Menschen zur Verzweiflung zu treiben und sie damit aufnahmebereit für den Messias zu machen - das erinnert zwar an Goethes Faust und an Mephisto als einen „Teil von jener Kraft, / Die stets das Böse will und stets das Gute schafft“, aber verglichen mit dem Goetheschen Teufel, der dem „Prolog im Himmel“ zufolge den zur Lethargie neigenden Menschen nur anstacheln soll, kommt dem Semael Zweigs eine entschiedener heilsgeschichtliche Funktion zu. Die Uraufführung ist in Berlin für 1914 geplant, wird dann aber wegen des Kriegsausbruchs abgesagt. Sie findet 1919 in Wien statt; in der Rolle des für die Handlung sehr wichtigen dreizehnjährigen Sohnes des Synagogendieners ist die junge Elisabeth Bergner zu sehen. - Die Umkehr des Abtrünnigen (1925, unter dem Titel Die Umkehr 1927) spielt Mitte des 18. Jahrhunderts in Brixen (Südtirol), in einzelnen Szenen auch in Podolien (Polen-Litauen), nämlich in der Heimat des Baalschem Rabbi Israel ben Elieser, des Begründers des Chassidismus. Der Bischof von Brixen leidet unter seiner seelischen Zerrissenheit, da er - als engagierter Feind der Juden - selbst von jüdischer Herkunft ist. Zur inneren Ruhe kommt er erst, als er nach einer Begegnung mit dem Baalschem sich zu seiner Herkunft bekennt, und zwar gegenüber einem Kardinal der katholischen Kirche, der, um Schaden von der Kirche abzuwenden, dem Bischof einen Ruhestand inkognito verschafft, statt die Wahrheit offenbar werden zu lassen - dies eine Pikanterie am Rande. Später legt Zweig im Übrigen eine umfangreiche Abhandlung vor, Juden auf der deutschen Bühne (1927), die die verschiedenen Bereiche des Theaters sorgsam mit einbezieht und die Juden als „Mitschöpfer“ des deutschen Theaters würdigt.

Ganz im biblischen Rahmen verbleibt ein mitten im Ersten Weltkrieg entstandenes Drama, das - in hohem Ton und zum Teil in Versen - sich zu einem spannenden Anti-Kriegsstück entwickelt, nämlich Stefan Zweigs Jeremias (1917): Der Prophet, von göttlichen Visionen bedrängt, warnt die Machthaber und das ausgesprochen wankelmütige Volk vor einem Krieg gegen Nabukadnezar, findet kein Gehör und spendet am Ende, nach der Niederlage und dem Fall Jerusalems, den Besiegten Trost mit seinem die Menschen mitreißenden Appell, im Glauben an „den Gott, der in ihnen lebt“, den Weg in die Zukunft und in die Ewigkeit anzutreten. Uraufgeführt wird das Stück 1918 in Zürich. 
Auf den geschichtlichen Stoff des württembergischen „Hoffaktors“ Josef Süß Oppenheimer aus dem 18. Jahrhundert greift Lion Feuchtwanger in seinem Drama Jud Süß zurück (1917; Neubearb. als Roman 1925). Süss (so Feuchtwangers Schreibung) ist verhasst, weil er zu Gunsten des Herzogs das Land auspresst. Als seine Tochter auf der Flucht vor den Nachstellungen des Herzogs tödlich verunglückt, rächt Süss sich am Herzog, indem er insgeheim dessen Vorhaben, den Katholizismus in Württemberg durchzusetzen, verrät und dadurch vereitelt. Der Herzog erleidet einen Schlaganfall. Süss, für die Taten des Herzogs verantwortlich gemacht, wird hingerichtet, nachdem er mehrere Bedingungen, unter denen er am Leben bleiben könnte, abgelehnt hat. Obwohl antijüdische Aversionen durchweg deutlich werden, geht es Feuchtwanger nicht lediglich um das Gegenüber von Juden und Christen, sondern auch um das von „Tat“ und „Verzicht“, wie er in verschiedenen Selbstkommentaren erklärt hat. Süss entwickelt sich, so gesehen, von einem Machtmenschen zu einem sich in Weisheit Drein-Ergebenden. $\mathrm{Zu}$ einer Figur des Übergangs wird er auch dadurch, dass ihm im zweiten Akt die Tatsache, dass sein Vater in Wahrheit ein Christ gewesen ist, von seiner jüdischen Mutter offenbart wird. Dass jedenfalls ein Votum gegen die Fixierung auf Macht und Tat im Jahr 1917 als ein Anti-Kriegs-Votum zu sehen ist, liegt auf der Hand.

Die Strömung der Neuen Sachlichkeit zieht in den 1920er Jahren natürlich auch die jüdischen Autoren in ihren Bann, ohne dass es dabei um jüdische Themen gehen muss. Ferdinand Bruckner zum Beispiel schreibt Krankheit der Jugend (1926), Die Verbrecher (1928) und Elisabeth von England (1930), das letztere Drama zu einem Zeitpunkt, zu dem historische Stoffe wieder vermehrt aufgegriffen werden. Friedrich Wolf liefert mit Cyankali (1929) mit Blick auf $\S 218$ einen wichtigen Beitrag zu der aktuellen Strafrechtsdebatte über das Thema der Abtreibung.

Wenn dann jedoch in der zweiten Hälfte der 1920er Jahre und später Dramen mit jüdischen Themen wieder vermehrt entstehen, ist darin eine Reaktion auf die zunehmende antisemitische Propaganda der Nationalsozialisten zu sehen. 1926 erscheint Franz Werfels Stück Paulus unter den Juden, das den Anfängen der Trennung zwischen Judentum und Christentum inmitten des Gegeneinanders einer Mehrzahl religiöser und politisch-nationaler Parteiungen nachspürt. Das Stück zeigt den Apostel Paulus, der, erschüttert von seinem Damaskus-Erlebnis, vergeblich versucht, seinen weithin geachteten alten Lehrer für das Christentum zu gewinnen, und dann seine Missionsreisen antritt. Einen signifikanten Tiefpunkt stellt am Ende des Stücks die Aufstellung des Bildes des römischen Kaisers Caligula als eines „Gottes“ im Tempel dar.

Hermann Ungars Stück Der rote General erscheint 1928, es spielt in der Zeit der Russischen Revolution 1917, und seine Hauptfigur Podkamjenski ist ein Porträt des Revolutionsführers Leo Trotzki. Podkamjenskis jüdische Herkunft ist durchgehend ein Thema und zeigt so das Fortleben antijüdischer Einstellungen auch unter den Revolutionären und damit die moralische Differenz zwischen dem - in idealisierter 
Zeichnung - ganz von sich selbst absehenden Podkamjenski und anderen eher robust-egozentrischen Revolutionären. Ermordet wird Podkamjenski am Ende von einem antisemitisch eingestellten zaristischen Offizier, der sich zum Anwalt der russischen Adelsgesellschaft und der russisch-orthodoxen Kirche aufwirft. Erfolgreich uraufgeführt wird das Stück mit Fritz Kortner in der Titelrolle.

1929 erscheint Walter Mehrings Stück Der Kaufmann von Berlin. Ein historisches Schauspiel aus der deutschen Inflation, das ein Panorama verschiedenster Themen und Motive sowie politischer und weltanschaulicher Positionen präsentiert. Im Zentrum steht der Ostjude Kaftan, der im Besitz von hundert Dollar 1923 nach Berlin gelangt, reich und mächtig wird und mit dem Ende der Inflation alles wieder verliert, nur scheinbar unterstützt durch einen in Wahrheit antisemitischen Rechtsanwalt, der einem nationalistischen Bund angehört und mit Freikorpsverbänden einen Putsch vorbereitet. Am Ende scheitert der Putsch, es kommt zu einem Pogrom im Berliner Scheunenviertel, nachdem schon durch das ganze Stück hindurch die Allgegenwart des Antisemitismus spürbar gewesen ist. Der Pogrom zeigt, dass „die deutsch-preußische Aufklärungs- und Akkulturationsgeschichte von eineinhalb Jahrhunderten an den grundsätzlichen Beziehungen zwischen Minorität und Majorität nichts geändert hat“ (Bayerdörfer 1993, 314). Das Stück führt 1929 in der Regie von Erwin Piscator zu einem der größten Theaterskandale der Weimarer Republik, obwohl die Inszenierung nicht eigentlich auf das „Rasseproblem“, sondern auf „ein Klassenproblem“ zielt (so Piscators eigene Worte).

Ebenfalls aus dem Jahr 1929 stammt Die Affäre Dreyfus von Hans José Rehfisch und Wilhelm Herzog, ein spannendes Stück, das einen kurzen Abschnitt, das Jahr 1898, aus der langjährigen Affäre (1894-1906) um den zu Unrecht wegen der Spionage für Deutschland verurteilten und schließlich rehabilitierten französischen Hauptmann Dreyfus herausgreift, den ersten Juden im französischen Generalstab. Gezeigt wird, wie gegen das antidemokratisch-reaktionär eingestellte Militär, seinerseits unterstützt durch eine im Volk verbreitete antijüdische Stimmung, erst allmählich die Wahrheit sich durchsetzt, für die sich insbesondere der Schriftsteller Émile Zola und der (historische) Oberstleutnant Picquart engagieren.

Paul Kornfeld, ehedem, wie erwähnt, ein Dramatiker des Expressionismus, legt 1930 seine Tragödie Jud Süss vor, die, abermals auf den historischen Stoff zurückgreifend, zunächst die Karriere eines pfiffig-zielstrebigen Aufsteigers zeigt. Dieser kennt sich nicht nur in Gelddingen aus, sondern er erscheint überraschenderweise auch in Fragen des Geschmacks - Kleidung, Architektur usw. - als ein Mann von Welt. Als ein Gegner ihn zu vergiften versucht, nimmt - dichterische Freiheit! versehentlich der Herzog das Gift zu sich. Nach dem Tod des Herzogs werden Süss Bestechungen und Betrügereien vorgeworfen; er wird verurteilt. Es handelt sich offensichtlich um ein Fehlurteil, denn Süss hat zwar durch die auf seinen Vorschlag hin eingerichteten neuen Steuern das Volk gegen sich aufgebracht, sich aber nicht in unlauterer Weise bereichert. Dafür dass der Rezipient dennoch nicht einseitig für 
ihn Partei ergreift, sorgt zwischenzeitlich eine Szene, in der Süss sich recht unfreundlich im Umgang mit jüdischen Bittstellern zeigt. In Leopold Jessners Inszenierung spielte Ernst Deutsch die Hauptrolle.

Else Lasker-Schülers Drama Arthur Aronymus und seine Väter (aus dem Jahr 1932) - mit dem Titelzusatz „aus meines geliebten Vaters Kinderjahren“ - stellt privat-familiengeschichtliche Momente in den historischen Kontext der antijüdischen Ausschreitungen im Jahr 1844 in Westfalen. Die (ihrem Ursprung nach nicht weiter erläuterte) wachsende antijüdische Stimmung wendet sich gegen die Familie des jüdischen Gutsbesitzers Moritz Schüler. Ein sogar drohender Pogrom wird schließlich abgewendet durch den katholischen Kaplan des Ortes und dessen vorgesetzten Bischof, die damit nicht die christliche Mehrheit vertreten. Mittels vieler Figuren jüdischer Herkunft, auch gesellschaftlicher Randfiguren, werden etliche Elemente des jüdischen Lebens in fünfzehn Bildern präsentiert, die dem Stück etwas Bilderbogenartiges vermitteln. Das Drama wirbt für ein einträchtiges Zusammenleben von Christen und Juden. Ein knappes Jahr nach seinem Erscheinen gelangen die Nationalsozialisten an die Macht. Die Uraufführung kommt daher erst im Dezember 1936 im Zürcher Schauspielhaus zustande; unter der Regie des Emigranten Leopold Lindtberg wirken etliche ebenfalls emigrierte Schauspieler mit.

\section{Literaturverzeichnis}

Angress, Ruth K.: „Wunsch- und Angstbilder. Jüdische Gestalten aus der deutschen Literatur des neunzehnten Jahrhunderts“. Schöne, Albrecht (Hrsg.): Kontroversen, alte und neue. Akten des VII. Internationalen Germanisten-Kongresses Göttingen 1986. Tübingen: Niemeyer, 1986, Bd. 1, 84-96.

Bayerdörfer, Hans-Peter: „,Harlekinade in jüdischen Kleidern?‘ Der szenische Status der Judenrollen zu Beginn des 19. Jahrhunderts“. Horch, Hans Otto; Denkler, Horst (Hrsg.): Conditio Judaica. Judentum, Antisemitismus und deutschsprachige Literatur vom 18. Jahrhundert bis zum Ersten Weltkrieg. 2. Teil. Tübingen: Niemeyer, 1989, 92-112.

Bayerdörfer, Hans-Peter: „Shylock in Berlin. Walter Mehring und das Judenporträt im Zeitstück der Weimarer Republik“. Horch, Hans Otto; Denkler, Horst (Hrsg.): Conditio Judaica. Judentum, Antisemitismus und deutschsprachige Literatur vom Ersten Weltkrieg bis 1933/1938. Tübingen: Niemeyer, 1993, 306-323.

Bayerdörfer, Hans-Peter; Fischer, Jens Malte (Hrsg.): Judenrollen. Darstellungsformen im europäischen Theater von der Restauration bis zur Zwischenkriegszeit. Tübingen: Niemeyer, 2008.

Beier, Nikolaj: „Die komödienhafte Inszenierung einer antisemitischen Affäre. Arthur Schnitzlers Professor Bernhardi“. Bayerdörfer, Hans-Peter; Fischer, Jens Malte (Hrsg.): Judenrollen. Darstellungsformen im europäischen Theater von der Restauration bis zur Zwischenkriegszeit. Tübingen: Niemeyer, 2008, 207-220.

Brauneck, Manfred: Die Welt als Bühne. Geschichte des europäischen Theaters. Bd. 3. Stuttgart, Weimar: Metzler, 1999.

Denkler, Horst: „,Lauter Juden‘. Zum Rollenspektrum der Juden-Figuren im populären Bühnendrama der Metternichschen Restaurationsperiode (1815-1848)“. Horch, Hans Otto; Denkler, Horst 
(Hrsg.): Conditio Judaica. Judentum, Antisemitismus und deutschsprachige Literatur vom 18. Jahrhundert bis zum Ersten Weltkrieg. 1. Teil. Tübingen: Niemeyer, 1988, 149-163.

Englhart, Andreas: „Ernst Tollers Stationendrama Die Wandlung auf der expressionistischen Experimentierbühne ,Die Tribüne““. Bayerdörfer, Hans-Peter; Fischer, Jens Malte (Hrsg.): Judenrollen. Darstellungsformen im europäischen Theater von der Restauration bis zur Zwischenkriegszeit. Tübingen: Niemeyer, 2008, 237-254.

Hein, Jürgen: „Judenthematik im Wiener Volkstheater“. Horch, Hans Otto; Denkler, Horst (Hrsg.): Conditio Judaica. Judentum, Antisemitismus und deutschsprachige Literatur vom

18. Jahrhundert bis zum Ersten Weltkrieg. 1. Teil. Tübingen: Niemeyer, 1988, 164-186.

Heine, Heinrich: Sämtliche Schriften. Hrsg. von Klaus Briegleb. Bd. 1. München: Hanser ${ }^{2} 1975$.

Horch, Hans Otto; Shedletzky, Itta: „Die deutsch-jüdische Literatur und ihre Geschichte“. Schoeps, Julius H. (Hrsg.): Neues Lexikon des Judentums. Gütersloh, München: Bertelsmann, 1992, 291-294.

Horch, Hans Otto: „Die unheilbar große Brüderkrankheit. Zum programmatischen Zeitgedicht Das neue Israelitische Hospital zu Hamburg von Heinrich Heine“. Bobinac, Marijan (Hrsg.): Literatur im Wandel. Festschrift für Viktor Žmegač zum 70. Geburtstag. Zagreb: Universität Zagreb, Abteilung für Germanistik der Philosophischen Fakultät, 1999, 41-52.

Kindermann, Heinz: Theatergeschichte Europas. Bd. 7. Salzburg: Müller, 1968.

Lea, Charlotte A.: Emancipation, Assimilation and Stereotype. The Image oft the Jew in German and Austrian Drama (1800-1850). Bonn: Bouvier, 1978.

Mittelmann, Hanni: „Expressionismus und Judentum. Horch, Hans Otto; Denkler, Horst (Hrsg.): Conditio Judaica. Judentum, Antisemitismus und deutschsprachige Literatur vom Ersten Weltkrieg bis 1933/1938. Tübingen: Niemeyer, 1993, 251-259.

Neubauer, Hans-Joachim: „Auf Begehr: Unser Verkehr. Über eine judenfeindliche Theaterposse im Jahre 1815“. Erb, Rainer; Schmidt, Michael (Hrsg.): Antisemitismus und jüdische Geschichte. Studien zu Ehren von Herbert A. Strauss. Berlin: Wissenschaftlicher Autorenverlag, 1987, 313-327.

Neubauer, Hans-Joachim: Judenfiguren. Drama und Theater im frühen 19. Jahrhundert. Frankfurt a.M., New York: Campus, 1994.

Richter, Matthias: Die Sprache jüdischer Figuren in der deutschen Literatur (1750-1933). Studien zu Form und Funktion. Göttingen: Wallstein, 1995.

Weissberg, Liliane: „Dramatic History. Reflections on a Biblical Play by Ludwig Robert”. Mendelsohn, Ezra (Hrsg.): Literary Strategies. Jewish Texts and Contexts. New York, Oxford: Oxford University Press, 1996, 3-20. 